\title{
A Thermomechanically Consistent Constitutive Theory for Modeling Micro-Void and/or Micro-Crack Driven Failure in Metals at Finite Strains
}

\author{
Celal Soyarslan \\ Institute of Continuum and Material Mechanics \\ Hamburg University of Technology \\ 21073 Hamburg, Germany \\ celal.soyarslan@tuhh.de \\ Ismail Cem Turtuk and Babur Deliktas \\ Department of Civil Engineering \\ Uludag̃ University, 16059 Bursa, Turkey \\ Swantje Bargmann \\ Institute of Continuum and Material Mechanics \\ Hamburg University of Technology \\ 21073 Hamburg, Germany \\ Institute of Materials Research \\ Helmholtz-Zentrum Geesthacht \\ 21502 Geesthacht, Germany
}

Received 24 June 2015

Revised 23 October 2015

Accepted 13 November 2015

Published 24 February 2016

\begin{abstract}
Within a continuum approximation, we present a thermomechanical finite strain plasticity model which incorporates the blended effects of micro-heterogeneities in the form of micro-cracks and micro-voids. The former accounts for cleavage-type of damage without any volume change whereas the latter is a consequence of plastic void growth. Limiting ourselves to isotropy, for cleavage damage a scalar damage variable $d \in[0,1]$ is incorporated. Its conjugate variable, the elastic energy release rate, and evolution law follow the formal steps of thermodynamics of internal variables requiring postulation of an appropriate damage dissipation potential. The growth of void volume fraction $f$ is incorporated using a Gurson-type porous plastic potential postulated at the effective stress space following continuum damage mechanics principles. Since the growth of micro-voids is driven by dislocation motion around voids the dissipative effects corresponding to the void growth are encapsulated in the plastic flow. Thus, the void volume fraction is used as a dependent variable using the conservation of mass. The predictive capability of the model is tested through uniaxial tensile tests at various temperatures $\Theta \in\left[-125^{\circ} \mathrm{C}\right.$, $125^{\circ} \mathrm{C}$. It is shown, via fracture energy plots, that temperature driven ductile-brittle
\end{abstract}

This is an Open Access article published by World Scientific Publishing Company. It is distributed under the terms of the Creative Commons Attribution 4.0 (CC-BY) License. Further distribution of this work is permitted, provided the original work is properly cited. 
transition in fracture mode is well captured. With an observed ductile-brittle transition temperature around $-50^{\circ} \mathrm{C}$, at lower temperatures fracture is brittle dominated by $d$ whereas at higher temperatures it is ductile dominated by $f$.

Keywords: Thermoplasticity; finite strain; void growth; cleavage; ductile-brittle transition.

\section{Introduction}

Mechanistically, ductile fracture is explained by nucleation, growth and coalescence of micro-voids. Brittle fracture, on the other hand, stems from the inter- or intragranular cleavage with micro-crack nucleation, growth and coalescence [Anderson, 2004; ASM International, 1996]. Both ductile and brittle fracture is observed in bcc metals. The transition from one mode to the other is mainly controlled by temperature $^{\mathrm{a}}$ : At lower temperatures fracture is brittle whereas at higher temperatures it is ductile.

One of the most widely used modeling approach for plasticity with microvoid growth is that of Gurson [1977]. Derived using homogenization over void-rigid plastic matrix aggregates and limit analysis, the yield potential of Gurson's plasticity model has hydrostatic stress dependence. A natural outcome of this formulation is irreversible volume change, i.e., plastic dilatation. This model is modified by Tvergaard and Needleman, by the introduction of void shape effects as well as acceleration in the void growth during void coalescence, to be named as GursonTvergaard-Needleman porous plasticity model [Tvergaard and Needleman, 1984] and by Chu and Needleman [1980] to account for void nucleation effects along with other contributors [Tvergaard, 1981, 1982a, 1982b; Needleman and Tvergaard, 1998; Nahshon and Hutchinson, 2008; Nahshon and Xue, 2009].

There are various modeling approaches for cleavage in metallic materials. The deterministic model by Ritchie-Knott-Rice [Ritchie et al., 1973] relies on a temperature and rate independent critical stress over the cleavage grain. Once the principal stresses averaged out over one or two grain-size region exceeds this threshold, the brittle fracture emanates. This threshold does not necessitate the presence of plastic flow. Incorporation of plastic flow is realized in Beremin's statistical model which, being based on the Weibull weakest link theory, reflects random nature of brittle fracture [Beremin, 1983; Mudry, 1987]. Another simple yet powerful approach to brittle fracture is the so-called energy limiters [Volokh, 2004, 2007, 2015]. By enforcing saturation in the strain energy function, energy limiters automatically bound stresses in the constitutive equations. Although useful, none of these models postulate an internal damage variable to account for gradual material deterioration by microcrack nucleation. Thus, dissipation associated with brittle fracture is not incorporated. Continuum damage mechanics remedies this gap by introducing scalar or tensorial damage variables and their conjugate variables which allows not only modeling of stiffness and strength degradation but also determination of inelastic

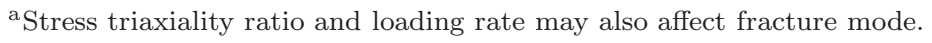


dissipation [Lemaitre, 1996; Lemaitre et al., 2013]. For the recent developments in brittle fracture modeling, we refer to Miehe et al. [2010, 2015], Andrieu et al. [2012] and Duda et al. [2015].

The present work introduces a thermodynamically consistent continuum approximation of micro-void and/or micro-crack driven failure at finite strains. To this end, in the spirit of Chaboche et al. [2006] we blend Gurson's porous plasticity model with Lemaitre's continuum damage mechanics. The blended constitutive model has two damage variables: the void volume fraction $f$ and the brittle damage variable $d$. Material fails by evolution of both damage sources. Since the growth of microvoids is driven by dislocation motion around voids, dissipative effects corresponding to the void growth are encapsulated in the plastic flow. Thus, the void volume fraction is a dependent variable using conservation of mass. For cleavage damage, a scalar variable is incorporated whose conjugate variable, the elastic energy release rate, and evolution law follow the formal steps of thermodynamics of internal variables requiring postulation of an appropriate damage dissipation potential. Hence, the brittle damage process is realistically reflected on a thermomechanically consistent ground. By these properties, the proposed model forms an appropriate basis for modeling fracture with ductile-brittle transitions driven by temperature or triaxiality.

Among various modeling attempts to the ductile-brittle transition problem in the literature [Shterenlikht, 2003; Hütter et al., 2014; Needleman and Tvergaard, 2000; Batra and Lear, 2004; Xia and Fong Shih, 1996], the most common one is based on a collective use of Gurson-type porous plasticity along with either RitchieKnott-Rice or Beremin model [Soyarslan et al., 2015; Needleman and Tvergaard, 2000; Hütter et al., 2014]. However, these approaches suffer from the mentioned inherent weaknesses pertaining to brittle fracture models. The noteworthy features of our work can be summarized as follows:

- Since our main motivation stems from modeling temperature driven ductilebrittle transition of fracture, unlike [Chaboche et al., 2006], we consider thermal coupling.

- Unlike Chaboche et al. [2006], we consider finite strains making use of a hyperelastic plastic type formulation relying on multiplicative decomposition of deformation gradient into recoverable and irrecoverable parts following [Håkansson et al., 2006].

- Although in the current study we limit ourselves to thermoelastic, plastic and damage isotropy, incorporation of brittle anisotropic damage is possible within the presented formalism.

- Using the kinematic coupling between plasticity and continuum damage, brittle damage evolution necessitates plastic flow which is consistent for metallic materials.

- Unlike cited ductile-brittle transition modeling approaches, our modeling approach accounts for gradual strength and stiffness loss as well as energy dissipation associated with brittle damage. 
- The model framework can also be exploited for fracture development under low triaxiality regimes for which Gurson's model is known to be ineffective.

\section{Fundamental Kinematics}

Let $\mathcal{B} \in \mathbb{R}^{3}$ be the material (initial), $\overline{\mathcal{B}} \in \mathbb{R}^{3}$ the intermediate and $\mathcal{B}_{t} \in \mathbb{R}^{3}$ the spatial (current) configurations of a body. ${ }^{\mathrm{b}}$ The motion of the body is described by a one-to-one nonlinear mapping $\varphi: \mathcal{B} \rightarrow \mathcal{B}_{t} \subset \mathbb{R}^{3}$. The deformation gradient $\mathbf{F}:=\partial \mathbf{x} \varphi$, is defined as the linear mapping of referential material tangents on their spatial counterparts, in which $\mathbf{X}$ defines the material coordinates in the reference configuration. The Jacobian of the deformation gradient is defined as $J=\operatorname{det}(\mathbf{F})=$ $\rho_{0} / \rho$, where $\rho_{0}$ and $\rho$ are densities at initial and spatial configurations, respectively. Multiplicative decomposition of the deformation gradient in recoverable $\mathbf{F}^{\mathrm{r}}$ and irrecoverable $\mathbf{F}^{\mathrm{p}}$ parts is postulated such that

$$
\mathbf{F}:=\mathbf{F}^{\mathrm{r}} \cdot \mathbf{F}^{\mathrm{p}}
$$

with $J^{\mathrm{r}}:=\operatorname{det}\left(\mathbf{F}^{\mathrm{r}}\right)$ and $J^{\mathrm{p}}:=\operatorname{det}\left(\mathbf{F}^{\mathrm{p}}\right)$. The recoverable part is associated with distortion of the crystal and the plastic part of the deformation gradient $\mathbf{F}^{\mathrm{p}}$ encapsulates the dislocation movements through its isochoric part [Håkansson et al., 2006]. $\mathbf{F}^{\mathrm{r}}$ is further partitioned into elastic and thermal parts using $\mathbf{F}^{\mathrm{r}}:=\mathbf{F}^{\mathrm{e}} \cdot \mathbf{F}^{\Theta}$. With this decomposition we introduce an additional imagined configuration $\hat{\mathcal{B}}$ which corresponds to a stress-free thermoplastic deformation where thermal expansion (or contraction) is driven by temperature change $\Delta \Theta=\Theta-\Theta_{0}$, where $\Theta$ and $\Theta_{0}$ represent the current and the initial temperature, respectively. The spatial velocity gradient is given by $\mathbf{l}:=\dot{\mathbf{F}} \cdot \mathbf{F}^{-1}$ which is additively decomposed into the rate of deformation tensor $\mathbf{d}=\operatorname{sym}(\mathbf{l})$ and the rate of $\operatorname{spin}$ tensor $\mathbf{w}=\operatorname{skw}(\mathbf{l})$ via $\mathbf{l}=\mathbf{d}+\mathbf{w}$, respectively. The recoverable and irrecoverable right Cauchy-Green deformation tensors are defined as $\mathbf{C}^{\mathrm{r}}=\left[\mathbf{F}^{\mathrm{r}}\right]^{\top} \cdot \mathbf{F}^{\mathrm{r}}$ and $\mathbf{C}^{\mathrm{p}}=\left[\mathbf{F}^{\mathrm{p}}\right]^{\top} \cdot \mathbf{F}^{\mathrm{p}}$, respectively. Similar partition, which proves convenient in the following developments, applies for $\mathbf{l}$ as well via $\mathbf{l}=\mathbf{l}^{\mathrm{r}}+\mathbf{l}^{\mathrm{p}}$ where $\mathbf{l}^{\mathrm{r}}:=\dot{\mathbf{F}}^{\mathrm{r}} \cdot\left[\mathbf{F}^{\mathrm{r}}\right]^{-1}$ and $\mathbf{l}^{\mathrm{p}}=\mathbf{F}^{\mathrm{r}} \cdot \mathbf{L}^{\mathrm{p}} \cdot\left[\mathbf{F}^{\mathrm{r}}\right]^{-1}$ with $\mathbf{L}^{\mathrm{p}}:=\dot{\mathbf{F}}^{\mathrm{p}} \cdot\left[\mathbf{F}^{\mathrm{p}}\right]^{-1}$. Letting recoverable logarithmic strain tensor defined by $\mathbf{e}_{\log }^{\mathrm{r}}:=\ln \left(\sqrt{\mathbf{C}^{\mathrm{r}}}\right)$, its partition into volumetric and isochoric parts reads $\mathbf{e}_{\log , \mathrm{vol}}^{\mathrm{r}}:=1 / 3 \ln J^{\mathrm{r}} \mathbf{1}=1 / 3 \operatorname{tr}\left(\mathbf{e}_{\log }^{\mathrm{r}}\right) \mathbf{1}$ and $\mathbf{e}_{\log , \text { iso }}^{\mathrm{r}}:=\ln \left(\sqrt{J^{\mathrm{r}-2 / 3} \mathbf{C}^{\mathrm{r}}}\right)=\operatorname{dev} \mathbf{e}_{\log }^{\mathrm{r}}$, respectively. Configurations and fundamental mappings are given in Fig. 1.

\footnotetext{
${ }^{\mathrm{b}}$ In this work, the following notations are used. Consistently assuming $\mathbf{a}, \mathbf{b}$, and $\mathbf{c}$ as three secondorder tensors, together with the Einstein's summation convention on repeated indices, $\mathbf{c}=\mathbf{a} \cdot \mathbf{b}$ represents the single contraction product with $c_{i k}=a_{i j} b_{j k} . d=\mathbf{a}: \mathbf{b}=a_{i j} b_{i j}$ represents the double contraction product, where $d$ is a scalar. $\mathbb{C}=\mathbf{a} \otimes \mathbf{b}$ represents tensor product with $C_{i j k l}=$ $a_{i j} b_{k l} \cdot \mathbf{a}^{\top}$ and $\mathbf{a}^{-1}$ denote the transpose and the inverse of $\mathbf{a}$, respectively $\partial_{\mathbf{a}} \mathbf{b}$ denotes the partial derivative of $\mathbf{b}$ with respect to $\mathbf{a}$, that is $\partial \mathbf{b} / \partial \mathbf{a} \cdot \operatorname{dev}(\mathbf{a})=\mathbf{a}-1 / 3 \operatorname{tr}(\mathbf{a}) \mathbf{1}$ and $\operatorname{tr}(\mathbf{a})=a_{i i}$ stand for the deviatoric part of and trace of a, respectively, $\mathbf{1}$ denoting the identity tensor. $\operatorname{sym}(\mathbf{a})$ and $\operatorname{skw}(\mathbf{a})$ denote symmetric and skew-symmetric parts of $\mathbf{a}$. a gives the material time derivative of $\mathbf{a}$. $\widehat{\mathbf{a}}$ is the rotationally neutralized representation of $\mathbf{a} .\langle x\rangle=1 / 2[x+|x|]$ describes the ramp function. Finally, div and grad denote the divergence and gradient operators with respect to the spatial configurations, respectively.
} 


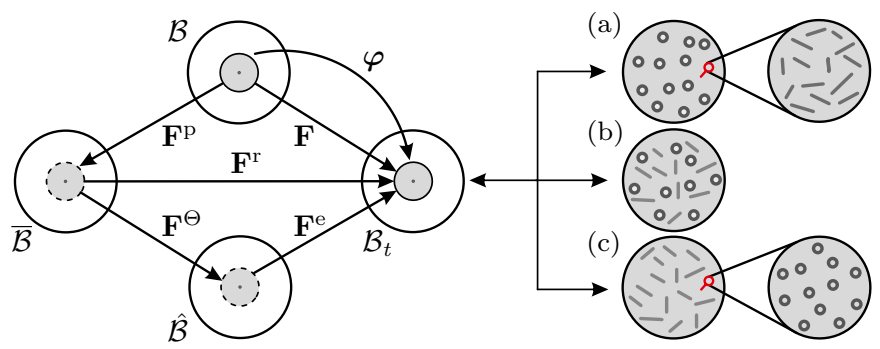

Fig. 1. On the left-hand side, the local multiplicative decomposition of the deformation gradient $\mathbf{F}$ into recoverable $\mathbf{F}^{\mathrm{r}}:=\mathbf{F}^{\mathrm{e}} \cdot \mathbf{F}^{\Theta}$ and irrecoverable $\mathbf{F}^{\mathrm{p}}$ parts and corresponding configurations are given. One the right-hand side, possible interpretations of the current framework are listed. (a) voids at upper scale and cracks at lower scale, (b) voids and cracks at identical scale, and (c) cracks at upper scale and voids at lower scale.

The assumption of a representative volume element with uniformly distributed spherical voids with a total volume of $d V^{\mathrm{v}}$ and plastically incompressible matrix with a total volume of $d V^{\mathrm{m}}$ with $d V^{\mathrm{v}}+d V^{\mathrm{m}}=d V$, lets one define the void volume fraction $f=d V^{\mathrm{v}} / d V$. Now, taking $J^{\mathrm{v}}=d V / d V^{\mathrm{m}}$ and letting the initial volume of the matrix be $d V^{\mathrm{m} 0}$ we define $J^{\mathrm{v} 0}=d V^{0} / d V^{\mathrm{m} 0}$. Since the matrix material is allowed to experience only thermoelastic dilatations we set $J^{\mathrm{r}}=d V^{\mathrm{m}} / d V^{\mathrm{m} 0}$. Thus, with $J=J^{\mathrm{r}} J^{\mathrm{p}}=d V / d V^{0}$ one has $J^{\mathrm{p}}=J^{\mathrm{v}} / J^{\mathrm{v} 0}$. Taking logarithms and the material time derivatives of both sides we reach $\overline{\ln \left(J^{\mathrm{p}}\right)}=\overline{\ln \left(J^{\mathrm{v}}\right)}$. Using $\overline{\ln \left(J^{\mathrm{p}}\right)}=\operatorname{tr}\left(\mathbf{L}^{\mathrm{p}}\right)$ one concludes that

$$
\dot{f}=[1-f] \operatorname{tr}\left(\mathbf{L}^{\mathrm{p}}\right) .
$$

Hence, being dependent on $\operatorname{tr}\left(\mathbf{L}^{\mathrm{p}}\right), f$ is part of the problem kinematics; in other words, the void growth is linked to the dislocation motion around the void. In this sense, it is not an independent state variable. On the other hand, the rather brittle micro-crack and micro-cleavage mechanisms are not fully accounted for by dislocation glide. Hence, in the current thermodynamic formalism, their treatment requires the introduction of a new state variable whose evolution necessitates postulation of an independent dissipation potential. To this end, continuum damage $d \in[0,1]$ phenomenologically reflects the softening response associated with accumulated micro-cracks. The interpretation of the envisioned framework is given on the right-hand side of Fig. 1. Without loss of generality, independent of the scale hierarchy depicted the void free matrix is plastically incompressible - hence, above definitions are valid.

\section{Thermodynamical Formulation}

\subsection{General theory}

Let $\psi$ denote the Helmholtz free energy per unit reference mass. Then, the first law of thermodynamics is expressed in reference configuration as

$$
\rho_{0} \Theta \dot{\eta}=\boldsymbol{\tau}: \mathbf{d}-\rho_{0} \dot{\Theta} \eta-\rho_{0} \dot{\psi}-J \operatorname{div} \mathbf{q}+\rho_{0} r
$$


where $\tau$ is the Kirchhoff stress tensor, $\Theta$ is the absolute temperature, $\eta$ is the entropy per unit mass, $\mathbf{q}$ is the spatial heat flux and $r$ is the body heat source per unit mass. With an abuse of notation, let $\psi=\psi(\mathcal{I})$, with $\mathcal{I}$ being the set of internal variables, the second law of thermodynamics supplies the following dissipation inequality represented in the reference configuration [Bargmann and Ekh, 2013]

$$
\mathcal{D}_{\text {loc }}=\boldsymbol{\tau}: \mathbf{d}-\rho_{0} \partial_{\mathcal{I}} \psi \cdot \dot{\mathcal{I}}-\frac{J}{\Theta} \mathbf{q} \cdot \operatorname{grad} \Theta \geq 0 .
$$

We further assume an additive decomposition into thermomechanical and conductive parts $\mathcal{D}_{\text {loc }}=\mathcal{D}_{\text {loc }}^{\text {thermech }}+\mathcal{D}_{\text {loc }}^{\text {con }}$ with

$$
\begin{gathered}
\mathcal{D}_{\text {loc }}^{\text {thermech }}:=\boldsymbol{\tau}: \mathbf{d}-\rho_{0} \partial_{\mathcal{I}} \psi \cdot \dot{\mathcal{I}} \geq 0, \\
\mathcal{D}_{\text {loc }}^{\text {cond }}:=-\frac{J}{\Theta} \mathbf{q} \cdot \operatorname{grad} \Theta \geq 0 .
\end{gathered}
$$

The latter inequality is satisfied by a generalized Fourier law adapted for the heat flux even in the presence of damage. The fulfillment of the former requires selection of the set $\mathcal{I}$ and its appropriate evolution laws. With $\mathcal{I}=\left\{\mathbf{C}^{\mathrm{r}}, d, \alpha, \Theta\right\}$, where $d$ is the scalar isotropic damage variable and $\alpha$ is the plastic hardening variable, Eq. (5.1) reads

$$
\begin{aligned}
\mathcal{D}_{\text {loc }}^{\text {thermech }}= & {\left[\boldsymbol{\tau}-2 \rho_{0}\left[\mathbf{F}^{\mathrm{r}}\right]^{\top} \cdot \partial_{\mathbf{C}^{\mathrm{r}}} \psi \cdot \mathbf{F}^{\mathrm{r}}\right]: \mathbf{d}^{\mathrm{r}}+\boldsymbol{\tau}: \mathbf{F}^{\mathrm{r}} \cdot \mathbf{L}^{\mathrm{p}} \cdot\left[\mathbf{F}^{\mathrm{r}}\right]^{-1} } \\
& -\left[\eta+\rho_{0} \partial_{\Theta} \psi\right] \dot{\Theta}-\rho_{0} \partial_{d} \psi \dot{d}-\rho_{0} \partial_{\alpha} \psi \dot{\alpha} \geq 0,
\end{aligned}
$$

with $\dot{\mathbf{C}}^{\mathrm{r}}=2\left[\mathbf{F}^{\mathrm{r}}\right]^{\top} \cdot \mathbf{d}^{\mathrm{r}} \cdot \mathbf{F}^{\mathrm{r}}$ and $\boldsymbol{\tau}: \mathbf{w}=0$ since $\mathbf{w}=-\mathbf{w}^{\top}$. The following state laws are derived in order for inequality (6) to be valid for arbitrary $\mathbf{d}^{\mathrm{r}}$ and $\dot{\Theta}$

$$
\begin{aligned}
\boldsymbol{\tau} & :=2 \rho_{0}\left[\mathbf{F}^{\mathrm{r}}\right]^{\top} \cdot \partial_{\mathbf{C}^{\mathrm{r}}} \psi \cdot \mathbf{F}^{\mathrm{r}}, \quad \beta:=\rho_{0} \partial_{\alpha} \psi, \\
Y & :=-\rho_{0} \partial_{d} \psi, \quad \eta:=-\rho_{0} \partial_{\Theta} \psi,
\end{aligned}
$$

where $Y$ and $\beta$ refer to the effective elastic energy release rate and the conjugate internal force variable of the plastic isotropic hardening. Substituting these back into the right-hand side of the inequality (6), one has the following reduced purely mechanical form

$$
\mathcal{D}_{\text {loc }}^{\text {red }}=\boldsymbol{\tau}: \mathbf{F}^{r} \cdot \mathbf{L}^{\mathrm{p}} \cdot \mathbf{F}^{r-1}+Y \dot{d}-\beta \dot{\alpha} \geq 0 .
$$

Let $\mathbf{M}:=\left[\mathbf{F}^{\mathrm{r}}\right]^{\top} \cdot \boldsymbol{\tau} \cdot\left[\mathbf{F}^{\mathrm{r}}\right]^{-\top}$ denote the Mandel stress tensor. With reference to the intermediate configuration one has $\boldsymbol{\tau}: \mathbf{F}^{\mathrm{r}} \cdot \mathbf{L}^{\mathrm{p}} \cdot\left[\mathbf{F}^{\mathrm{r}}\right]^{-1}=\mathbf{M}: \mathbf{L}^{\mathrm{p}}$ and Eq. (8) can be rearranged to give

$$
\mathcal{D}_{\text {loc }}^{\text {red }}=\mathbf{M}: \mathbf{L}^{\mathrm{p}}-\beta \dot{\alpha}+Y \dot{d} \geq 0 .
$$

This clearly depicts the work-conjugacy of the plastic part of the velocity gradient $\mathbf{L}^{\mathrm{p}}$ defined at intermediate configuration and the Mandel stress tensor $\mathbf{M}$. With the assumption of elastic isotropy, one has $\left[\mathbf{F}^{\mathrm{r}}\right]^{\top} \cdot \boldsymbol{\tau} \cdot\left[\mathbf{F}^{\mathrm{r}}\right]^{-\top}=\left[\mathbf{R}^{\mathrm{r}}\right]^{\top} \cdot \boldsymbol{\tau} \cdot \mathbf{R}^{\mathrm{r}}$, where $\mathbf{R}^{\mathrm{r}}$ is the elastic rotation found using the polar decomposition of the recoverable deformation gradient $\mathbf{F}^{\mathrm{r}}=\mathbf{R}^{\mathrm{r}} \cdot \mathbf{U}^{\mathrm{r}}$ with $\mathbf{U}^{\mathrm{r}}=\sqrt{\mathbf{C}^{\mathrm{r}}}$ representing the recoverable 
stretch tensor. Hence, in the subsequent developments we use $\mathbf{M}=\left[\mathbf{R}^{\mathrm{r}}\right]^{\top} \cdot \boldsymbol{\tau} \cdot \mathbf{R}^{\mathrm{r}}$. That is, $\mathbf{M}$ amounts to the rotated Kirchhoff stress tensor. Hence, both $\mathbf{M}$ and $\boldsymbol{\tau}$ share identical invariants which gains importance while postulating the plastic potential.

Finally, introducing the dissipation potential $\Upsilon$ as

$$
\Upsilon(\mathbf{M}, \beta, Y, d, f):=\phi^{\mathrm{p}}(\mathbf{M}, \beta ; d, f)+\phi^{\mathrm{d}}(Y ; d),
$$

and applying the generalized normality rule one derives the following evolution equations which satisfy the positiveness of the dissipation along with loading/unloading conditions $\lambda \geq 0, \phi^{\mathrm{p}} \leq 0, \lambda \phi^{\mathrm{p}}=0$

$$
\begin{gathered}
\mathbf{L}^{\mathrm{p}}=\lambda \partial_{\mathbf{M}} \Upsilon=\lambda \partial_{\mathbf{M}} \phi^{\mathrm{p}}, \\
\dot{\alpha}=-\lambda \partial_{\beta} \Upsilon=-\lambda \partial_{\beta} \phi^{\mathrm{p}}, \\
\dot{d}=\lambda \partial_{Y} \Upsilon=\lambda \partial_{Y} \phi^{\mathrm{d}},
\end{gathered}
$$

where $\lambda$ is the plastic multiplier. This way, through a kinematic coupling, we link evolution of brittle damage with plastic flow. This condition expresses the fact that brittle failure can only occur when plastic deformation occurs which is an appropriate assumption for cleavage in metallic materials [Beremin, 1983]. On the contrary, Ritchie-Knott-Rice criterion for brittle fracture, for instance, does not require plastic flow [Ritchie et al., 1973].

\subsection{Specification of the constitutive forms}

The choice of the form of the Helmholtz free energy function $\psi$ constitutes the basis in deriving the constitutive equations for the representative volume element. In the current treatment, an additively decomposed Helmholtz free energy into elastic, plastic and thermal parts is selected via $\psi=\psi^{\mathrm{e}}+\psi^{\mathrm{p}}+\psi^{\Theta}$. For the elastic part we further apply a volumetric deviatoric split using $\psi^{\mathrm{e}}=\psi_{\mathrm{vol}}^{\mathrm{e}}+\psi_{\text {iso }}^{\mathrm{e}}$ and, following a Lemaitre-type formalism, we state couple micro-crack damage only with thermoelasticity using

$$
\begin{aligned}
\rho_{0} \psi_{\text {vol }}^{\mathrm{e}} & =[1-d] \kappa\left[\frac{1}{2}\left[\operatorname{tr}\left(\mathbf{e}_{\log }^{\mathrm{r}}\right)\right]^{2}-3 \alpha_{\Theta}\left[\Theta-\Theta_{0}\right] \operatorname{tr}\left(\mathbf{e}_{\log }^{\mathrm{r}}\right)\right], \\
\rho_{0} \psi_{\text {iso }}^{\mathrm{e}} & =[1-d] \mu\left[\operatorname{dev} \mathbf{e}_{\mathrm{log}}^{\mathrm{r}}: \operatorname{dev} \mathbf{e}_{\mathrm{log}}^{\mathrm{r}}\right],
\end{aligned}
$$

in the reference configuration. $\kappa=E / 3[1-2 \nu]$ and $\mu=E / 2[1+\nu]$ are the elastic bulk modulus and shear modulus, respectively with $E$ representing the modulus of elasticity and $\nu$ denoting the elastic Poisson's ratio. For the plastic part we have

$$
\rho_{0} \psi^{\mathrm{p}}=\left[1-f_{0}\right]\left[\tau_{\mathrm{m}, \infty}-\tau_{\mathrm{m}, 0}\right]\left[\alpha+\frac{1}{\omega_{\mathrm{m}}}\left[\exp \left(-\omega_{\mathrm{m}} \alpha\right)-1\right]\right]
$$

in the reference configuration. Hence, the isotropic plastic hardening of the representative volume element is taken to obey an exponential behavior, where $\tau_{\mathrm{m}, 0}$ and 
$\tau_{\mathrm{m}, \infty}$ denote the initial and saturated Kirchhoff-type matrix yield stresses and $\omega_{\mathrm{m}}$ is the matrix hardening saturation rate. The link between the material matrix and the representative volume element is reflected in the factor $\left[1-f_{0}\right]$. The reason of using $\left[1-f_{0}\right]$ rather than $[1-f]$ is due to the Kirchhoff stress based hardening formulation which complies with the space of the postulated yield function [Håkansson et al., 2006].

Using the transformation $\boldsymbol{\tau}=\left[\mathbf{R}^{\mathrm{r}}\right]^{\top} \cdot \mathbf{M} \cdot \mathbf{R}^{\mathrm{r}}$, the plastic potential $\phi^{\mathrm{p}}(\mathbf{M}, \beta ; d, f)$ can be reparametrized to give $\phi^{\mathrm{p}}(\overline{\boldsymbol{\tau}}, \beta, f)$ with the effective stress definition $\overline{\boldsymbol{\tau}}=$ $\tau /[1-d]$. Based on an analysis of a single spherical void in a spherical shell presented in Gurson [1977] where the change of void shape is neglected, the effectively isotropic yield function $\phi^{\mathrm{p}}$, representing approximate form for the yield surface of a randomly voided solid containing a volume fraction $f$ of voids is then defined as

$$
\phi^{\mathrm{p}}(\overline{\boldsymbol{\tau}}, \beta, f):=\frac{1}{2 \tau_{\mathrm{m}}}\left[\bar{\tau}_{\mathrm{eq}}^{2}+2 f \tau_{\mathrm{m}}^{2} \cosh \left(\frac{\operatorname{tr} \overline{\boldsymbol{\tau}}}{2 \tau_{\mathrm{m}}}\right)-\tau_{\mathrm{m}}^{2}\left[1+f^{2}\right]\right] \leq 0,
$$

where $\bar{\tau}_{\text {eq }}$ is introduced as the effective equivalent Kirchhoff stress through $\bar{\tau}_{\text {eq }}=$ $\sqrt{3 / 2[\operatorname{dev} \overline{\boldsymbol{\tau}}: \operatorname{dev} \overline{\boldsymbol{\tau}}]}$. Note that $\bar{\tau}_{\mathrm{eq}}=\bar{M}_{\mathrm{eq}}:=\sqrt{3 / 2[\operatorname{dev} \overline{\mathbf{M}}: \operatorname{dev} \overline{\mathbf{M}}]}$ as well as $\operatorname{tr} \boldsymbol{\tau}=\operatorname{tr} \mathbf{M}$. The matrix flow stress is represented by $\tau_{\mathrm{m}}=\tau_{\mathrm{m}, 0}+\beta_{\mathrm{m}}$ with $\beta_{\mathrm{m}}=\beta /\left[1-f_{0}\right]$ denoting the matrix hardening.

Remark 1. For fully developed plastic flow, i.e., $\phi^{\mathrm{p}}(\overline{\boldsymbol{\tau}}, \beta)=0$, under traceless stress state with $\operatorname{tr} \overline{\boldsymbol{\tau}} / 2 \tau_{\mathrm{m}} \rightarrow 0$ one has $\cosh \left(\operatorname{tr} \overline{\boldsymbol{\tau}} / 2 \tau_{\mathrm{m}}\right) \rightarrow 1$. Hence, $\bar{\tau}_{\text {eq }}^{2}=[1-f]^{2} \tau_{\mathrm{m}}^{2}$, which can be rearranged, using the effective stress definition, to give

$$
\tau_{\text {eq }}=[1-d][1-f] \tau_{\mathrm{m}} .
$$

This depicts the effect of two distinct damage sources on the material response and the link between the fictitious effective material subscale free of defects and the mesoscopic behavior as depicted in the scenarios given in Fig. 1.

Remark 2. In the spirit of Håkansson et al. [2006], the motivation for the evolution laws is considered in the absence of micro-voids, however, with micro-cracks. Accordingly, the flow potential reduces to $\phi^{\mathrm{p}}(\overline{\boldsymbol{\tau}}, \beta)=\bar{\tau}-\tau_{m} \leq 0$. Following associative plasticity, the evolution laws for $\mathbf{L}^{\mathrm{p}}$ and $\alpha$ are governed by the normality rule

$$
\mathbf{L}^{\mathrm{p}}=\lambda \frac{\partial \phi^{\mathrm{p}}}{\partial \mathbf{M}}=\frac{\lambda}{1-d} \frac{3}{2} \frac{\operatorname{dev} \overline{\mathbf{M}}}{\tau_{\mathrm{m}}} \quad \text { and } \quad \dot{\alpha}=-\lambda \frac{\partial \phi^{\mathrm{p}}}{\partial \beta}=\lambda .
$$

Defining the equivalent plastic strain rate as $\dot{\epsilon}^{\mathrm{p}}=\sqrt{2 / 3 \mathbf{L}^{\mathrm{p}}: \mathbf{L}^{\mathrm{p}}}$ and using $\dot{\alpha}=\lambda$ one reaches $\dot{\epsilon}^{\mathrm{p}}=\dot{\alpha} /[1-d]$. Hence, the rate of plastic work $w^{\mathrm{p}}$ in the reference configuration reads

$$
w^{\mathrm{p}}=\operatorname{dev} \mathbf{M}: \mathbf{L}^{\mathrm{p}}=[1-d] \tau_{\mathrm{m}} \dot{\epsilon}^{\mathrm{p}} .
$$


Thus, in comparison to Håkansson et al. [2006], the effect of the cleavage damage is also involved in the work equivalence relation.

In the light of the Remark 2, and following Håkansson et al. [2006], we postulate the validity of the following definitions for the porous plasticity model as well

$$
\begin{aligned}
\dot{\alpha} & =\lambda, \\
\dot{\epsilon}^{\mathrm{p}} & =\frac{\dot{\alpha}}{1-d} .
\end{aligned}
$$

Hence Eq. (15a) is replaced with Eq. (11b) for the definition of $\dot{\alpha}$ in the foregoing developments. We also postulate the following generalization of the plastic work equivalence

$$
\left[1-f_{0}\right][1-d] \dot{\epsilon}^{\mathrm{p}} \tau_{\mathrm{m}}=\mathbf{M}: \mathbf{L}^{\mathrm{p}},
$$

which gains importance in definition of the total mechanical dissipation. Note that for a Cauchy-type matrix yield stress $\sigma_{\mathrm{m}}=1 / J^{\mathrm{r}} \tau_{\mathrm{m}}$ one has $\left[1-f_{0}\right][1-d] \dot{\epsilon}^{\mathrm{p}} \tau_{\mathrm{m}}=$ $1 / J[1-f][1-d] \dot{\epsilon}^{\mathrm{p}} \sigma_{\mathrm{m}}$.

Due to the dependence of $\phi^{\mathrm{p}}$ on the trace of the stress tensor, $\mathbf{L}^{\mathrm{p}}$ is not traceless. Consequently, plastic dilatant effects are incorporated. Hence, the void volume fraction is associated with the plastic dilatant strains through the mass balance relation $\dot{f}=[1-f] \operatorname{tr}\left(\mathbf{L}^{\mathrm{p}}\right)$, also given in Eq. (2). Thus, unlike the isotropic continuum damage variable $d$, one does not need an additional dissipation potential in evaluation of the void volume fraction rate since it is plastic flow that accounts for the dissipation associated. The rate of $d$, on the other hand, is derived using a dissipation potential using Eq. (11c). $\phi^{\mathrm{d}}$ is selected to take the form of Lemaitre-type damage dissipation potential [Lemaitre, 1996]

$$
\phi^{\mathrm{d}}(Y)=\frac{S}{[1+m][1-d]^{n}}\left[\frac{\left\langle Y-Y_{0}\right\rangle}{S}\right]^{m+1},
$$

where $m, n, S$ and $Y_{0}$ are associated material parameters. $Y_{0}$ represents the threshold value of strain energy release rate governing the initiation of cleavage damage.

Now, using Eqs. (7) along with the defined potentials in Eqs. (12) and (13), we arrive at explicit forms of the conjugate variables $\mathbf{M}, \beta$ and $Y$ as

$$
\begin{aligned}
\mathbf{M} & =[1-d]\left[\kappa \operatorname{tr}\left(\mathbf{e}_{\log }^{\mathrm{r}}\right) \mathbf{1}+2 \mu \operatorname{dev} \mathbf{e}_{\log }^{\mathrm{r}}-3 \kappa \alpha_{\Theta}\left[\Theta-\Theta_{0}\right] \mathbf{1}\right], \\
\beta & =\left[1-f_{0}\right]\left[\tau_{\mathrm{m}, \infty}-\tau_{\mathrm{m}, 0}\right]\left[1-\exp \left(-\omega_{\mathrm{m}} \alpha\right)\right], \\
Y & =\kappa\left[\frac{1}{2}\left[\operatorname{tr}\left(\mathbf{e}_{\log }^{\mathrm{r}}\right)\right]^{2}-3 \alpha_{\Theta}\left[\Theta-\Theta_{0}\right] \operatorname{tr}\left(\mathbf{e}_{\log }^{\mathrm{r}}\right)\right]+\mu\left[\operatorname{dev} \mathbf{e}_{\log }^{\mathrm{r}}: \operatorname{dev} \mathbf{e}_{\log }^{\mathrm{r}}\right],
\end{aligned}
$$

where the matrix hardening is represented with $\beta_{\mathrm{m}}=\beta /\left[1-f_{0}\right]=\left[\tau_{\mathrm{m}, \infty}-\tau_{\mathrm{m}, 0}\right][1-$ $\left.\exp \left(-\omega_{\mathrm{m}} \alpha\right)\right]$.

For the plastic flow and the evolution of $d$, we use Eqs. (11a) and (11c) along with the dissipation potential components given in Eqs. (14) and (17), respectively. 
The hardening variable rate $\dot{\alpha}$ obeys Eq. (15a). Collectively, the evolution equations read

$$
\begin{aligned}
\mathbf{L}^{\mathrm{p}} & =\frac{\lambda}{1-d}\left[\frac{3}{2} \frac{\operatorname{dev} \overline{\mathbf{M}}}{\tau_{\mathrm{m}}}-\frac{1}{2} f \sinh \left(\frac{q_{2} \operatorname{tr} \overline{\mathbf{M}}}{2 \tau_{\mathrm{m}}}\right) \mathbf{1}\right], \\
\dot{\alpha} & =\lambda, \quad \dot{d}=\frac{\lambda}{[1-d]^{n}}\left[\frac{\left\langle Y-Y_{0}\right\rangle}{S}\right]^{m},
\end{aligned}
$$

where $\overline{\mathbf{M}}=\mathbf{M} /[1-d]$. Now, substituting Eqs. (18) and (19) into the right-hand side of Eq. (9) together with using Eq. (16), the mechanical dissipation reads

$$
\mathcal{D}_{\text {loc }}^{\text {red }}=\left[1-f_{0}\right][1-d] \dot{\epsilon}^{\mathrm{p}} \tau_{\mathrm{m}, 0}+\dot{\epsilon}^{\mathrm{p}} \frac{1}{[1-d]^{n-1}} Y\left[\frac{\left\langle Y-Y_{0}\right\rangle}{S}\right]^{m} .
$$

The second term reflects the dissipative effect of cleavage-type damage which is not accounted for in Håkansson et al. [2006]. Equation (20) is always nonnegative, hence, the second law of thermodynamics is fulfilled. For convenience, a summary of the constitutive model is given in BOX $\mathbf{1}$.

BOX 1. A summary of the proposed model for general 3D stress-state.

(1) Multiplicative kinematics

$$
\mathbf{F}=\mathbf{F}^{\mathrm{r}} \cdot \mathbf{F}^{\mathrm{p}} \quad \text { and } \quad \mathbf{F}^{\mathrm{r}}=\mathbf{F}^{\mathrm{e}} \cdot \mathbf{F}^{\Theta}
$$

(2) Thermoelastic stress-strain relationship

$$
\mathbf{M}=[1-d]\left[\kappa \operatorname{tr}\left(\mathbf{e}_{\log }^{\mathrm{r}}\right) \mathbf{1}+2 \mu \operatorname{dev} \mathbf{e}_{\log }^{\mathrm{r}}-3 \kappa \alpha_{\Theta}\left[\Theta-\Theta_{0}\right] \mathbf{1}\right] .
$$

(3) State laws for hardening and damage conjugate variables

$$
\begin{aligned}
\beta & =\left[1-f_{0}\right]\left[\tau_{\mathrm{m}, \infty}-\tau_{\mathrm{m}, 0}\right]\left[1-\exp \left(-\omega_{\mathrm{m}} \alpha\right)\right], \\
Y & =\kappa\left[\frac{1}{2}\left[\operatorname{tr}\left(\mathbf{e}_{\log }^{\mathrm{r}}\right)\right]^{2}-3 \alpha_{\Theta}\left[\Theta-\Theta_{0}\right] \operatorname{tr}\left(\mathbf{e}_{\log }^{\mathrm{r}}\right)\right]+\mu\left[\operatorname{dev} \mathbf{e}_{\log }^{\mathrm{r}}: \operatorname{dev} \mathbf{e}_{\log }^{\mathrm{r}}\right] .
\end{aligned}
$$

(4) Matrix yield stress and hardening

$$
\tau_{\mathrm{m}}=\tau_{\mathrm{m}, 0}+\beta_{\mathrm{m}} \quad \text { and } \quad \beta_{\mathrm{m}}=\frac{\beta}{1-f_{0}} .
$$

(5) Thermoelastic domain in stress space (single surface)

$$
\mathbb{E}_{\boldsymbol{\tau}}=\left\{[\overline{\boldsymbol{\tau}}, \beta, f] \in \mathbb{S} \times \mathbb{R}^{+} \times \mathbb{R}^{+}: \phi^{\mathrm{p}}(\overline{\boldsymbol{\tau}}, \beta, f) \leq 0\right\},
$$

where $\mathbb{S}$ represents the vector space of symmetric second order tensors and

$$
\phi^{\mathrm{p}}(\overline{\boldsymbol{\tau}}, \beta, f)=\frac{1}{2 \tau_{\mathrm{m}}}\left[\bar{\tau}_{\mathrm{eq}}^{2}+2 f \tau_{\mathrm{m}}^{2} \cosh \left(\frac{\operatorname{tr} \overline{\boldsymbol{\tau}}}{2 \tau_{\mathrm{m}}}\right)-\tau_{\mathrm{m}}^{2}\left[1+f^{2}\right]\right] .
$$

(Continued) 
(6) Flow rule (associative model)

$$
\mathbf{L}^{\mathrm{p}}=\frac{\lambda}{1-d}\left[\frac{3}{2} \frac{\operatorname{dev} \overline{\mathbf{M}}}{\tau_{\mathrm{m}}}-\frac{1}{2} f \sinh \left(\frac{\operatorname{tr} \overline{\mathbf{M}}}{2 \tau_{\mathrm{m}}}\right) \mathbf{1}\right] .
$$

(7) Evolution equations for hardening, damage and porosity

$$
\begin{aligned}
& \dot{\alpha}=\lambda \quad \text { and } \quad \dot{\epsilon}^{\mathrm{p}}=\frac{\lambda}{1-d}, \\
& \dot{d}=\frac{\lambda}{[1-d]^{n}}\left[\frac{\left\langle Y-Y_{0}\right\rangle}{S}\right]^{m}, \\
& \dot{f}=[1-f] \operatorname{tr}\left(\mathbf{L}^{\mathrm{p}}\right) .
\end{aligned}
$$

(8) Kuhn-Tucker loading/unloading (complementarity) conditions

$$
\lambda \geq 0, \quad \phi^{\mathrm{p}}(\overline{\boldsymbol{\tau}}, \beta, f) \leq 0, \quad \lambda \phi^{\mathrm{p}}(\overline{\boldsymbol{\tau}}, \beta, f)=0 .
$$

(9) Consistency condition

$$
\lambda \dot{\phi}^{\mathrm{p}}(\overline{\boldsymbol{\tau}}, \beta, f)=0 .
$$

\section{Application - Uniaxial Tension}

In this section we first present a systematic reduction of the theory to monotonic uniaxial tension. Solution of the resultant reduced equation set allows conduction of some parametric studies which show predictive capabilities of the model.

\subsection{Specification of equations for uniaxial tensile state of stress}

Let $\mathbf{e}_{1}, \mathbf{e}_{2}$ and $\mathbf{e}_{3}$ denote orthogonal unit vectors associated with $x$-, $y$ - and $z$ directions. We are interested in tensile loading in $x$-direction where the material motion is free in $y$ - and $z$-directions. This corresponds to a uniaxial state of stress and triaxial state of strain with

$$
\begin{aligned}
\boldsymbol{\tau} & =\tau \mathbf{e}_{1} \otimes \mathbf{e}_{1} \\
\mathbf{F} & =\lambda_{1} \mathbf{e}_{1} \otimes \mathbf{e}_{1}+\lambda_{2}\left[\mathbf{e}_{2} \otimes \mathbf{e}_{2}+\mathbf{e}_{3} \otimes \mathbf{e}_{3}\right],
\end{aligned}
$$

where $\lambda_{1}$ and $\lambda_{2}$ represent principal stretches in $x$ - and $y$ - (equivalently $z$-) directions. With Eq. (21a) following equivalences hold: $\tau_{\text {eq }}=\tau=\operatorname{tr} \tau$. Also, in absence of rotation, i.e., $\mathbf{R}^{r}=\mathbf{1}$, one has $\boldsymbol{\tau} \equiv \mathbf{M}$. Using Eq. (21b) the Jacobian of the deformation gradient becomes $J=\lambda_{1} \lambda_{2}^{2}$. In analogy to Eq. (21b), the recoverable and irrecoverable parts of the deformation gradient can be given as

$$
\begin{aligned}
& \mathbf{F}^{\mathrm{r}}=\lambda_{1}^{\mathrm{r}} \mathbf{e}_{1} \otimes \mathbf{e}_{1}+\lambda_{2}^{\mathrm{r}}\left[\mathbf{e}_{2} \otimes \mathbf{e}_{2}+\mathbf{e}_{3} \otimes \mathbf{e}_{3}\right], \\
& \mathbf{F}^{\mathrm{p}}=\lambda_{1}^{\mathrm{p}} \mathbf{e}_{1} \otimes \mathbf{e}_{1}+\lambda_{2}^{\mathrm{p}}\left[\mathbf{e}_{2} \otimes \mathbf{e}_{2}+\mathbf{e}_{3} \otimes \mathbf{e}_{3}\right] .
\end{aligned}
$$




\section{Soyarslan et al.}

The recoverable part given in Eq. (22a) is further decomposed into the elastic and thermal parts viz. $\mathbf{F}^{\mathrm{r}}=\mathbf{F}^{\mathrm{e}} \cdot \mathbf{F}^{\Theta}$, where, with the assumption of thermally isotropic material $\mathbf{F}^{\Theta}$ is defined as a spherical tensor with $\mathbf{F}^{\Theta}:=\lambda^{\Theta} \mathbf{1}$. Accordingly, in the given orthogonal triad one has

$$
\begin{aligned}
& \mathbf{F}^{\mathrm{e}}=\lambda_{1}^{\mathrm{e}} \mathbf{e}_{1} \otimes \mathbf{e}_{1}+\lambda_{2}^{\mathrm{e}}\left[\mathbf{e}_{2} \otimes \mathbf{e}_{2}+\mathbf{e}_{3} \otimes \mathbf{e}_{3}\right], \\
& \mathbf{F}^{\Theta}=\lambda^{\Theta}\left[\mathbf{e}_{1} \otimes \mathbf{e}_{1}+\mathbf{e}_{2} \otimes \mathbf{e}_{2}+\mathbf{e}_{3} \otimes \mathbf{e}_{3}\right] .
\end{aligned}
$$

Hence, the right Cauchy-Green deformation tensor $\mathbf{C}=\mathbf{F}^{\top} \cdot \mathbf{F}$ computes

$$
\mathbf{C}=\left[\lambda_{1}^{\mathrm{e}}\right]^{2}\left[\lambda_{1}^{\mathrm{p}}\right]^{2}\left[\lambda^{\Theta}\right]^{2} \mathbf{e}_{1} \otimes \mathbf{e}_{1}+\left[\lambda_{2}^{\mathrm{e}}\right]^{2}\left[\lambda_{2}^{\mathrm{p}}\right]^{2}\left[\lambda^{\Theta}\right]^{2}\left[\mathbf{e}_{2} \otimes \mathbf{e}_{2}+\mathbf{e}_{3} \otimes \mathbf{e}_{3}\right] .
$$

Now, using the definition of the logarithmic strain $\mathbf{e}_{\log }=1 / 2 \log \mathbf{C}$ we reach

$$
\mathbf{e}_{\log }=\mathbf{e}_{\log }^{\mathrm{e}}+\mathbf{e}_{\log }^{\mathrm{p}}+\mathbf{e}_{\log }^{\Theta},
$$

where

$$
\begin{aligned}
& \mathbf{e}_{\log }^{\mathrm{e}}=\log \lambda_{1}^{\mathrm{e}} \mathbf{e}_{1} \otimes \mathbf{e}_{1}+\log \lambda_{2}^{\mathrm{e}}\left[\mathbf{e}_{2} \otimes \mathbf{e}_{2}+\mathbf{e}_{3} \otimes \mathbf{e}_{3}\right], \\
& \mathbf{e}_{\log }^{\mathrm{p}}=\log \lambda_{1}^{\mathrm{p}} \mathbf{e}_{1} \otimes \mathbf{e}_{1}+\log \lambda_{2}^{\mathrm{p}}\left[\mathbf{e}_{2} \otimes \mathbf{e}_{2}+\mathbf{e}_{3} \otimes \mathbf{e}_{3}\right], \\
& \mathbf{e}_{\log }^{\Theta}=\log \lambda^{\Theta}\left[\mathbf{e}_{1} \otimes \mathbf{e}_{1}+\mathbf{e}_{2} \otimes \mathbf{e}_{2}+\mathbf{e}_{3} \otimes \mathbf{e}_{3}\right] .
\end{aligned}
$$

Equation (25) encapsulates an additive decomposition in logarithmic strains carried out from a multiplicative decomposition of the deformation gradient. For convenience, we use the notation $e_{\log }^{\mathrm{e}}=\log \lambda_{1}^{\mathrm{e}}, e_{\log }^{\mathrm{p}}=\log \lambda_{1}^{\mathrm{p}}$ and $e_{\log }^{\Theta}=\log \lambda^{\Theta}$. Letting $\lambda^{\Theta}:=\exp \int_{\Theta_{0}}^{\Theta} \alpha_{\Theta}(\theta) d \theta$ and $\alpha_{\Theta}(\Theta)$ represent the linearc thermal expansion coefficient, omitting the dependence of $\alpha_{\Theta}$ on the temperature we find $J^{\Theta}:=\operatorname{det}\left(\mathbf{F}^{\Theta}\right)=$ $\left[\lambda^{\Theta}\right]^{3}=\exp \left(3 \alpha_{\Theta}\left[\Theta-\Theta_{0}\right]\right)$ where $\lambda^{\Theta}=\exp \left(\alpha_{\Theta}\left[\Theta-\Theta_{0}\right]\right)$. Thus $e_{\log }^{\Theta}=\alpha_{\Theta}\left[\Theta-\Theta_{0}\right]$. The elastic stress definition given in Eq. (18) and the vanishing stress components in $y$ - and $z$-directions reveal $\log \lambda_{2}^{\mathrm{e}}=-\nu \log \lambda_{1}^{\mathrm{e}}$ and supplies

$$
\tau=[1-d] E\left[e_{\log }^{\mathrm{r}}-\alpha_{\Theta}\left[\Theta-\Theta_{0}\right]\right]
$$

Also the total recoverable logarithmic strain tensor $\mathbf{e}_{\log }^{\mathrm{r}}=\mathbf{e}_{\log }^{\mathrm{e}}+\mathbf{e}_{\log }^{\Theta}$ reads

$$
\mathbf{e}_{\log }^{\mathrm{r}}=\left[e_{\log }^{\mathrm{e}}+e_{\log }^{\Theta}\right] \mathbf{e}_{1} \otimes \mathbf{e}_{1}+\left[-\nu e_{\log }^{\mathrm{e}}+e_{\log }^{\Theta}\right]\left[\mathbf{e}_{2} \otimes \mathbf{e}_{2}+\mathbf{e}_{3} \otimes \mathbf{e}_{3}\right],
$$

for which the volumetric and the deviatoric parts are computed as

$$
\begin{aligned}
\operatorname{tr} \mathbf{e}_{\log }^{\mathrm{r}} & =[1-2 \nu] e_{\log }^{\mathrm{e}}+3 e_{\log }^{\Theta}, \\
\operatorname{dev} \mathbf{e}_{\log }^{\mathrm{r}} & =\frac{2}{3}[1+\nu] e_{\log }^{\mathrm{e}} \mathbf{e}_{1} \otimes \mathbf{e}_{1}-\frac{1}{3}[1+\nu] e_{\log }^{\mathrm{e}}\left[\mathbf{e}_{2} \otimes \mathbf{e}_{2}+\mathbf{e}_{3} \otimes \mathbf{e}_{3}\right] .
\end{aligned}
$$

Using Eq. (29) along with Eq. (18c) gives the elastic energy release rate $e_{\log }^{\mathrm{e}}$ as

$$
Y=\frac{1}{2}\left[E\left[e_{\log }^{\mathrm{e}}\right]^{2}-9 \kappa\left[\alpha_{\Theta}\right]^{2}\left[\Theta-\Theta_{0}\right]^{2}\right],
$$

${ }^{\mathrm{c}}$ The volumetric thermal expansion coefficient, on the other hand, amounts for $3 \alpha_{\Theta}(\Theta)$ for thermally isotropic materials. 
or in terms of $e_{\log }^{\mathrm{r}}$ as

$$
Y=\frac{1}{2} E\left[e_{\log }^{\mathrm{r}}\right]^{2}-E e_{\log }^{\mathrm{r}} \alpha_{\Theta}\left[\Theta-\Theta_{0}\right]-3 \kappa[1+\nu]\left[\alpha_{\Theta}\right]^{2}\left[\Theta-\Theta_{0}\right]^{2} .
$$

Finally, using $\mathbf{L}^{p}:=\dot{\mathbf{F}}^{p} \cdot\left[\mathbf{F}^{p}\right]^{-1}$ we have

$$
\mathbf{L}^{\mathrm{p}}=\frac{\dot{\lambda}_{1}^{\mathrm{p}}}{\lambda_{1}^{\mathrm{p}}} \mathbf{e}_{1} \otimes \mathbf{e}_{1}+\frac{\dot{\lambda}_{2}^{\mathrm{p}}}{\lambda_{2}^{\mathrm{p}}}\left[\mathbf{e}_{2} \otimes \mathbf{e}_{2}+\mathbf{e}_{3} \otimes \mathbf{e}_{3}\right] \equiv \dot{\mathbf{e}}^{\mathrm{p}} .
$$

For convenience, the complete reformulation of the model under uniaxial tension is given in BOX 2. The rate equations together with the given yield function form a differential-algebraic equation set which is solved semi-explicitly. To this end, the vector of unknowns is represented by $\mathbf{x}=\{\tau, \alpha, f\}$. Assuming $\mathbf{x}=\mathbf{x}(\Delta \lambda)$, where $\Delta \lambda$ denotes the incremental plastic multiplier, the yield function is reformulated as a nonlinear function of $\Delta \lambda$ with $\phi^{\mathrm{p}}(\mathbf{x}(\Delta \lambda))$. Applying Taylor series expansion to $\phi^{\mathrm{p}}$ with backward-Euler integration of the unknowns leads to an iterative solution with $\Delta \lambda^{\langle k+1\rangle}=\Delta \lambda^{\langle k\rangle}-\left[\phi^{\mathrm{p}}\right]^{\langle k\rangle} /\left[\partial \phi^{\mathrm{p}} / \partial \mathbf{x} \cdot \partial \mathbf{x} / \partial \Delta \lambda\right]^{\langle k\rangle}$ where $\langle k\rangle$ represents the iteration index. The iterations are run under a desired tolerance where unknowns updates are realized at each iteration. The integration of $d$ is realized subsequent to the convergence in a staggered manner using the converged incremental plastic multiplier.

BOX 2. A reduction of the proposed model to monotonic uniaxial tension.

(1) Additive kinematics

$$
e_{\log }=e_{\log }^{\mathrm{r}}+e_{\log }^{\mathrm{p}} \quad \text { and } \quad e_{\log }^{\mathrm{r}}=e_{\log }^{\mathrm{e}}+e_{\log }^{\Theta},
$$

where $e_{\log }^{\Theta}=\alpha_{\Theta}\left[\Theta-\Theta_{0}\right]$.

(2) Thermoelastic stress-strain relationship

$$
\tau=[1-d] E\left[e_{\log }^{\mathrm{r}}-\alpha_{\Theta}\left[\Theta-\Theta_{0}\right]\right] .
$$

(3) State laws for hardening and damage conjugate variables

$$
\begin{aligned}
\beta & =\left[1-f_{0}\right]\left[\tau_{\mathrm{m}, \infty}-\tau_{\mathrm{m}, 0}\right]\left[1-\exp \left(-\omega_{\mathrm{m}} \alpha\right)\right], \\
Y & =\frac{1}{2} E\left[e_{\log }^{\mathrm{r}}\right]^{2}-E e_{\log }^{\mathrm{r}} \alpha_{\Theta}\left[\Theta-\Theta_{0}\right]-3 \kappa[1+\nu]\left[\alpha_{\Theta}\right]^{2}\left[\Theta-\Theta_{0}\right]^{2} .
\end{aligned}
$$

(4) Matrix yield stress and hardening

$$
\tau_{\mathrm{m}}=\tau_{\mathrm{m}, 0}+\beta_{\mathrm{m}} \quad \text { and } \quad \beta_{\mathrm{m}}=\frac{\beta}{1-f_{0}} .
$$

(5) Thermoelastic domain in stress space (single surface)

$$
\mathbb{E}_{\tau}=\left\{[\bar{\tau}, \beta, f] \in \mathbb{R}^{+} \times \mathbb{R}^{+} \times \mathbb{R}^{+}: \phi^{\mathrm{p}}(\bar{\tau}, \beta, f) \leq 0\right\},
$$

(Continued) 
where

$$
\phi^{\mathrm{p}}(\bar{\tau}, \beta, f)=\frac{1}{2 \tau_{\mathrm{m}}}\left[\bar{\tau}^{2}+2 f \tau_{\mathrm{m}}^{2} \cosh \left(\frac{\bar{\tau}}{2 \tau_{\mathrm{m}}}\right)-\tau_{\mathrm{m}}^{2}\left[1+f^{2}\right]\right] .
$$

(6) Flow rule (associative model)

$$
\dot{e}_{\log }^{\mathrm{p}}=\frac{\lambda}{1-d}\left[\frac{\bar{\tau}}{\tau_{\mathrm{m}}}-\frac{1}{2} f \sinh \left(\frac{\bar{\tau}}{2 \tau_{\mathrm{m}}}\right)\right] .
$$

(7) Evolution equations for hardening, damage and porosity

$$
\begin{aligned}
& \dot{\alpha}=\lambda \quad \text { and } \quad \dot{\epsilon}^{\mathrm{p}}=\frac{\lambda}{1-d}, \\
& \dot{d}=\frac{\lambda}{[1-d]^{n}}\left[\frac{\left\langle Y-Y_{0}\right\rangle}{S}\right]^{m}, \\
& \dot{f}=\frac{3}{2} \frac{\lambda[1-f]}{1-d} f \sinh \left(\frac{\bar{\tau}}{2 \tau_{\mathrm{m}}}\right) .
\end{aligned}
$$

(8) Kuhn-Tucker loading/unloading (complementarity) conditions

$$
\lambda \geq 0, \quad \phi^{\mathrm{p}}(\bar{\tau}, \beta, f) \leq 0, \quad \lambda \phi^{\mathrm{p}}(\bar{\tau}, \beta, f)=0 .
$$

(9) Consistency condition

$$
\lambda \dot{\phi}^{\mathrm{p}}(\bar{\tau}, \beta, f)=0
$$

\subsection{Results and discussions}

Our derivations correspond to a single material point loaded under uniaxial stress state. Hence, there is no heat conduction. We also disregard the heat generation by dissipative inelastic processes and conduct simulations at constant ten different temperatures $\Theta_{1} \cdots \Theta_{10}$ of equal temperature steps from $-125^{\circ} \mathrm{C}$ to $125^{\circ} \mathrm{C}$ where $\Theta_{1}=-125^{\circ} \mathrm{C}$ and $\Theta_{10}=125^{\circ} \mathrm{C}$. All analyses start from a stress-free configuration. Since $T=T_{0}$ throughout the loading, no thermal strains occur. Moreover, during loading the stress triaxiality ratio $\eta$ defined as $\eta:=\operatorname{tr} \tau / 3 \tau_{\text {eq }}$ is constant and equal to $1 / 3$. Due to lack of sufficient stress triaxiality, void growth occurs slowly. In order to accelerate void growth and obtain realistic failure strains we select a rather high initial void volume content with $f_{0}=0.055$. Moreover, following Tvergaard and Needleman [Tvergaard and Needleman, 1984], $f$ in the yield function definition given in Eq. (14) is replaced by $f^{*}$ which encapsulates acceleration of the void growth during the void coalescence via

$$
f^{*}(f)=f+\left\langle f-f_{\mathrm{c}}\right\rangle \frac{f_{\mathrm{u}}^{*}-f_{\mathrm{f}}}{f_{\mathrm{f}}-f_{\mathrm{c}}} .
$$

Here, $f_{\mathrm{c}}$ and $f_{\mathrm{f}}$ describe the critical void volume fraction at incipient coalescence and the fraction at failure, respectively. Finally, $f_{\mathrm{u}}^{*}=1 / q_{1}$. For thermal properties 
Table 1. Selected steel-like material parameters.

\begin{tabular}{lccc}
\hline Parameter & Symbol & Value & Unit \\
\hline Thermal expansion coef. & $\alpha_{\Theta}$ & $10^{-5}$ & $\mathrm{~K}^{-1}$ \\
Melting temperature & $\Theta_{\text {melt }}$ & 1717 & $\mathrm{~K}$ \\
Reference temperature & $\Theta_{\mathrm{ref}}$ & 298 & $\mathrm{~K}$ \\
Young's modulus & $E$ & 210 & $\mathrm{GPa}$ \\
Poisson's ratio & $\nu$ & 0.3 & {$[-]$} \\
Matrix hardening & $\tau_{\mathrm{m}, \infty}$ & 1000 & $\mathrm{MPa}$ \\
& $\tau_{\mathrm{m}, 0}$ & 500 & $\mathrm{MPa}$ \\
& $\omega_{\mathrm{m}}$ & 15 & {$[-]$} \\
Gurson model & $f_{0}$ & 0.055 & {$[-]$} \\
& $f_{\mathrm{c}}$ & 0.075 & {$[-]$} \\
& $f_{\mathrm{f}}$ & 0.10 & {$[-]$} \\
& $f_{\mathrm{u}}^{*}$ & 1 & {$[-]$} \\
Lemaitre model & $Y_{0}$ & 3 & $\mathrm{MPa}$ \\
& $m$ & 1 & {$[-]$} \\
& $n$ & 2 & {$[-]$} \\
& $S$ & 0.25 & $\mathrm{MPa}$ \\
\hline
\end{tabular}

and matrix hardening, steel-like but hypothetical parameters are selected. For convenience, Table 1 compiles the material parameters used at reference temperature $\Theta_{\text {ref }}=25^{\circ} \mathrm{C}$.

The temperature effect on the matrix yield stress is adapted using data available in the literature [Ritchie et al. 1973]. Accordingly, defining homologous temperature $\Omega$ with $\Omega:=\left[\Theta-\Theta_{\text {ref }}\right] /\left[\Theta_{\text {melt }}-\Theta_{\text {ref }}\right]$, we compute the matrix yield stress as $\beta_{\mathrm{m}}(\Omega)=t_{y}(\Omega) \beta_{\mathrm{m}}$ where $t_{y}(\Omega)=1846 \Omega^{4}-520.36 \Omega^{3}+50.422 \Omega^{2}-1.9124 \Omega+1$. Figure 2 depicts the variation of $t_{y}$ as a function of $\Theta$.

Two conditions are used in decision of the material failure: In the first one local instability condition is considered with $d \tau / d \epsilon<0$, whereas in the second one

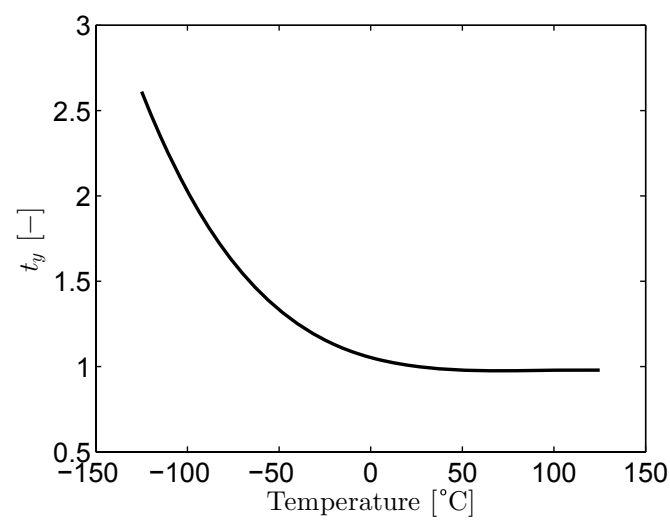

Fig. 2. Temperature effect on the yield stress as a scaling factor adapted from Ritchie et al. [1973]. Room temperature is taken as $25^{\circ} \mathrm{C}$ at which the yield stress factor is unity. Beyond room temperature there occurs only a slight decrease within the range of selected temperature interval. The curve is represented by a continuous function of the homologous temperature $\Omega$ with $t_{y}(\Omega)=1846 \Omega^{4}-520.36 \Omega^{3}+50.422 \Omega^{2}-1.9124 \Omega+1$. 


\section{Soyarslan et al.}

a sufficient loss of the stress bearing capacity of the material point is taken into account with $\tau<$ TOL where TOL $=0.01 \times \tau_{\mathrm{m}, 0}$. The former could be seen as a consequence of Drucker's stability postulate or more elaborate discontinuous bifurcation analyses which locates the initiation of localization as a precursor to fracture through the vanishing determinant of the acoustic tensor. For both failure criteria the absorbed energy to failure, i.e., the area under the stress-strain curves, are computed and plotted for corresponding temperatures. The results are shown in Figs. 3 and 4 . In each figure the stress, damage and void volume fraction plots as a function of the axial logarithmic strain are given for $\Theta_{1} \cdots \Theta_{10}$. Also the failure energy plots are given. The stress plots for both criteria show that at higher temperatures plastic strains are considerably larger than those at lower temperatures. It is seen that, in agreement with the presented results of Doghri [1995], the accumulation of

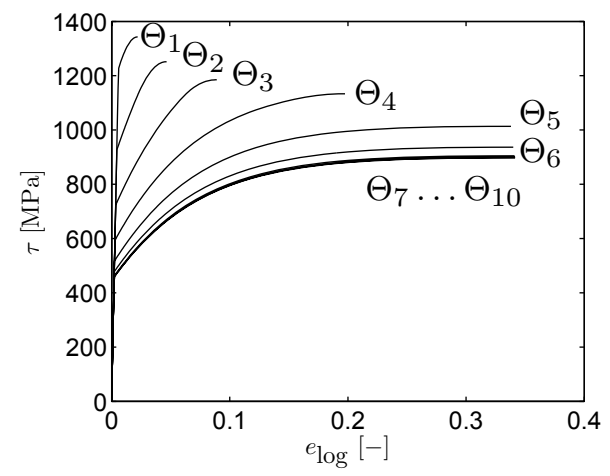

(a)

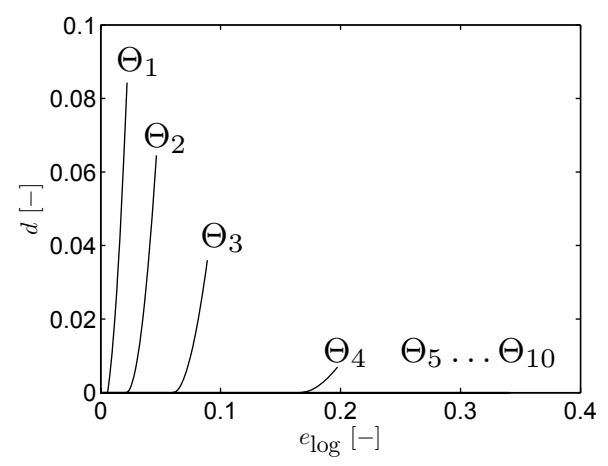

(c)

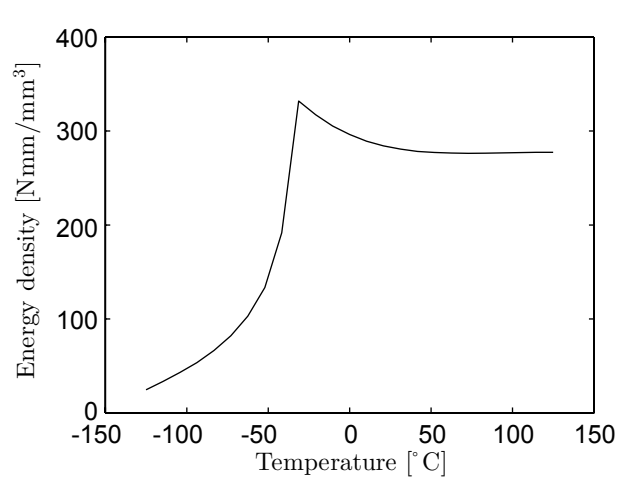

(b)

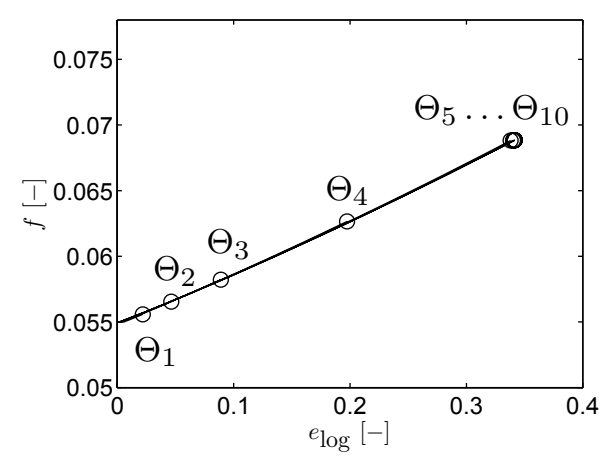

(d)

Fig. 3. Results for criterion $d \tau / d e_{\log }<0$ at 10 different temperatures $\Theta_{1} \cdots \Theta_{10}$ of equal temperature steps from $-125^{\circ} \mathrm{C}$ to $125^{\circ} \mathrm{C}$, where $\Theta_{1}=-125^{\circ} \mathrm{C}$ and $\Theta_{10}=125^{\circ} \mathrm{C}$ : (a) stress-strain curves, (b) energy per unit (reference) volume computed using $\int \tau d e_{\text {log }}$, as seen a sharp ductilebrittle transition is observed in energy density demand until neck where the ductile-brittle transition temperature is around $-50^{\circ} \mathrm{C}$, (c) damage $d$ accumulation until neck, and (d) void volume fraction $f$ accumulation until neck. The markers show $f$ values reached at each temperature. 


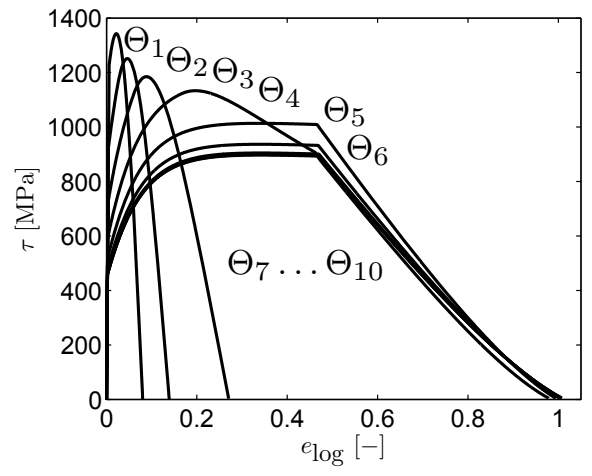

(a)

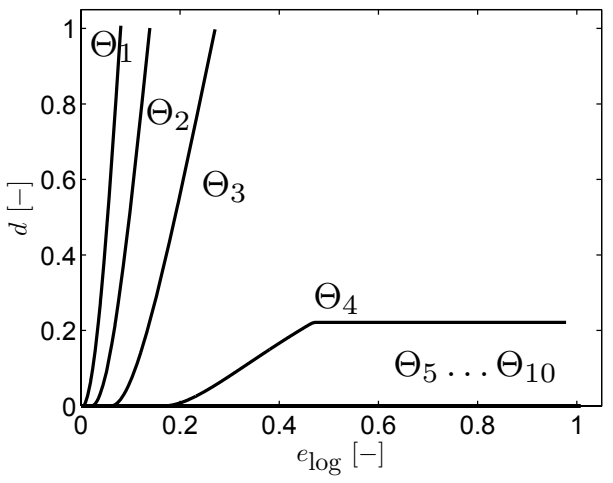

(c)

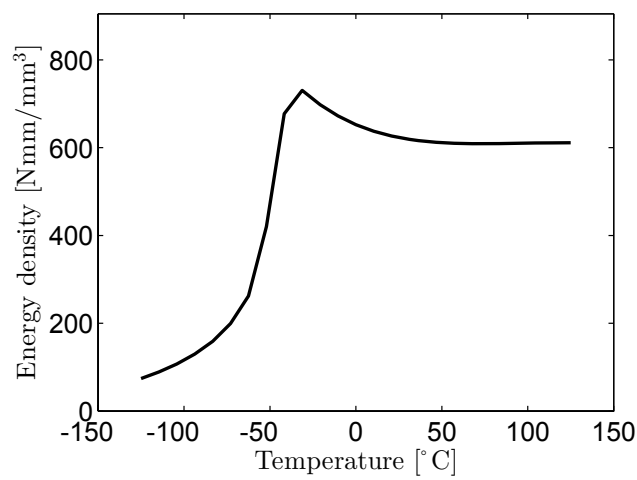

(b)

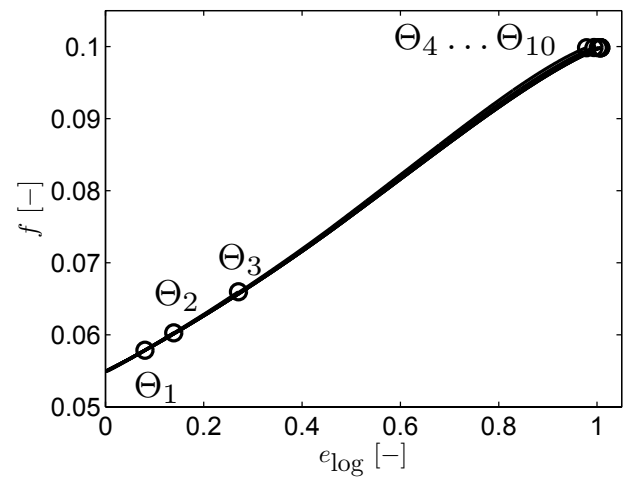

(d)

Fig. 4. Results for criterion $\tau<$ TOL where TOL $=0.01 \times \tau_{\mathrm{m}, 0}$ at 10 different temperatures $\Theta_{1} \cdots \Theta_{10}$ of equal temperature steps from $-125^{\circ} \mathrm{C}$ to $125^{\circ} \mathrm{C}$, where $\Theta_{1}=-125^{\circ} \mathrm{C}$ and $\Theta_{10}=$ $125^{\circ} \mathrm{C}$ : (a) stress-strain curves, (b) energy per unit (reference) volume computed using $\int \tau d e_{\log }$, as seen a sharp ductile-brittle transition is observed in energy density demand until complete loss of load carrying capacity where the ductile-brittle transition temperature is around $-50^{\circ} \mathrm{C},(\mathrm{c})$ damage $d$ accumulation until neck, and $(\mathrm{d})$ void volume fraction $f$ accumulation until neck. The markers show $f$ values reached at each temperature.

damage components $d$ and $f$ at the point of stability loss are lower than that of loss of complete load carrying capacity. Note that for due to lack of sufficient void volume fraction accumulation for $\Theta_{1} \cdots \Theta_{3}$ with the criterion $\tau<$ TOL $\times \tau_{\mathrm{m}, 0}, d$ reaches close to its theoretical limit $d=1$. The occurrence of a kink and subsequent rather accelerated drop of the stress response for increased temperatures is due to the enforced void coalescence condition.

It is notable that, although a drastic difference is observed in continuum damage variable evolution for different temperatures the void volume fraction is less sensitive to the yield value changes. Two main differences are observed in $d$ evolution curves for different temperatures: First the damage initiation strains and second the damage evolution rates. The former is due to the fact that the evolution of 
$d$ requires the damage driving force $Y$ reach the threshold $Y_{0}$. Higher yield stress at lower temperatures allows fulfillment of this requirement easily. At higher temperatures, i.e., $\Theta_{5} \cdots \Theta_{10}, Y$ never reaches $Y_{0}$, and thus $d$ ceases to evolve. Then, the whole softening mechanism is controlled by the void growth, hence failure is ductile. With the same token, higher $Y$ observed at lower temperatures results in an increased rate of damage evolution. Then, the whole softening mechanism is controlled by the evolution of $d$, hence failure is brittle.

In both material failure assumptions, the failure energy density plots show a sharp increase in the energy demand with temperature around $-50^{\circ} \mathrm{C}$. Hence, the desired ductile-brittle transition in the failure mode was possible where $-50^{\circ} \mathrm{C}$ point amounts for the ductile-brittle transition temperature. The energy demand until complete stress loss is slightly over double the energy demand until neck, but the main characteristics of the curve is not changed. There results show that the proposed framework proves useful in modeling temperature driven ductile-brittle transition of the fracture mode in metallic materials.

\section{Conclusion}

A theoretical framework for coupled porous thermoplasticity and continuum damage mechanics has been formulated preserving isotropy, within a thermodynamic consistency at finite strains. Multiplicative split of the deformation gradient in elastic, plastic and thermal parts has been utilized as a basis for finite strain kinematics. The framework aims at modeling blended mechanisms of simultaneous microvoid and micro-crack driven material degradation. Hence, the building blocks are selected as the Gurson-type porous plasticity model and Lemaitre-type continuum damage mechanics. Considering the possible application of the proposed model as the ductile-to-brittle transition of the fracture mode, the main advantage that the proposed model shows over the widely-used framework (e.g., porous plasticity for ductile failure and Ritchie-Knott-Rice or Beremin local approaches to cleavage) is that the energy dissipation associated with the cleavage-type of fracture is accounted for within the formalism of continuum damage mechanics. The isotropic damage variable $d$ introduced via Lemaitre formalism is able to take care of the strength and stiffness degradation related to the micro-cracks without volumetric changes, whereas void volumetric changes are encapsulated in the evolution of the void volume fraction $f$. The evolution of $d$ is formulated by a damage dissipation potential which devises the energy release rate $Y_{0}$ as a threshold for cleavage damage to initiate at the process zone. The evolution of $f$ follows the mass conservation relation which does not necessitate an additional dissipation potential. Extension of the model to anisotropic cleavage damage is possible through a fully intermediate configuration formulation of the yield function. The predictive capability of the model is demonstrated through application problems assuming uniaxial state of stress. It is shown by absorbed energy plots for different temperatures that for the presented 
parameter set remedying the limited stress triaxiality conditions, the model predicts the temperature driven ductile-brittle transition of fracture mode sufficiently well.

\section{Acknowledgment}

Financial support for this work provided by the German Science Foundation (DFG) under contract PAK 250 (TP4) is gratefully acknowledged.

\section{References}

Anderson, T. [2004] Fracture Mechanics: Fundamentals and Applications (CRC Press, Boca Raton).

Andrieu, A., Pineau, A., Besson, J., Ryckelynck, D. and Bouaziz, O. [2012] "Bimodal Beremin-type model for brittle fracture of inhomogeneous ferritic steels: Theory and applications," Engineering Fracture Mechanics 95, 84-101.

ASM International [1996] Handbook Vol. 19: Fatigue and Fracture (ASM International).

Batra, R. and Lear, M. [2000] "Simulation of brittle and ductile fracture in an impact loaded prenotched plate," International Journal of Fracture 126, 179-203.

Bargmann, S. and Ekh, M. [2013] "Microscopic temperature field prediction during adiabatic loading in a gradient extended crystal plasticity theory," International Journal of Solids and Structures 50, 899-906.

Beremin, F. [1983] "A local criterion for cleavage fracture of a nuclear pressure vessel steel," Metallurgical Transactions 14A, 2277-2287.

Chaboche, J., Boudifa, M. and Saanouni, K. [2006] "A CDM approach of ductile damage with plastic compressibility," International Journal of Fracture 137, 51-75.

Chu, C. C. and Needleman, A. [1980] "Void nucleation effects in biaxially stretched sheets," Journal of Engineering Materials and Technology 102, 249-256.

Doghri, I. [1995] "Numerical implementation and analysis of a class of metal plasticity models coupled with ductile damage," International Journal for Numerical Methods in Engineering 38, 3403-3431.

Duda, F. P., Ciarbonetti, A., Sanchez, P. J. and Huespe, A. E. [2015] "A phase field/gradient damage model for brittle fracture in elastic-plastic solids," International Journal of Plasticity 65, 269-296.

Gurson, A. [1977] "Continuum theory of ductile rupture by void nucleation and growth Part I. Yield criteria and rules for porous ductile media," Journal of Engineering Materials and Technology 99, 2-15.

Håkansson, P., Wallin, M. and Ristinmaa, M. [2006] "Thermomechanical response of nonlocal porous material," International Journal of Plasticity 22, 2066-2090.

Hütter, G., Linse, T., Roth, S., Mühlich, U. and Kuna, M. [2014] "A modelling approach for the complete ductile-to-brittle transition region: Cohesive zone in combination with a non-local Gurson-model," International Journal of Fracture 185, 129-153.

Lemaitre, J. [1996] A Course on Damage Mechanics (Springer-Verlag, Berlin).

Lemaitre, J., Desmorat, R. and Sauzay, M. [2013] "Anisotropic damage law of evolution," European Journal of Mechanics and Solids 19, 187-208.

Itskov, M. [2004] "On the application of the additive decomposition of generalized strain measures in large strain plasticity," Mechanics Research Communications 31, 507-517.

Miehe, C., Welschinger, F. and Hofacker, M. [2010] "Thermodynamically consistent phasefield models of fracture: Variational principles and multi-field FE implementations," International Journal for Numerical Methods in Engineering 83, 1273-1311. 
Miehe, C., Hofacker, M., Schaenzel, L.-M. and Aldakheel, F. [2015] "Phase field modeling of fracture in multi-physics problems. Part II. Coupled brittle-to-ductile failure criteria and crack propagation in thermo-elastic-plastic solids," Computer Methods in Applied Mechanics Engineering 294, 486-522.

Mudry, F. [1987] "A local approach to cleavage fracture," Nuclear Engineering and Design 105, 65-76.

Needleman, A. and Tvergaard, V. [1998] "Dynamic crack growth in a nonlocal progressively cavitating solid," European Journal of Mechanics A/Solids 17(3), 421-438.

Needleman, A. and Tvergaard, V. [2000] "Numerical modeling of the ductile-brittle transition," International Journal of Fracture 101, 73-97.

Nahshon, K. and Hutchinson, J. [2008] "Modification of the Gurson model to shear failure," European Journal of Mechanics A/Solids 27, 1-17.

Nahshon, K. and Xue, Z. [2009] "A modified Gurson model and its applications to punchout experiments," Engineering Fracture Mechanics 76, 997-1009.

Ritchie, R., Knott, J. and Rice, J. [1973] "On the relationship between critical tensile stress and fracture toughness in mild steel," Journal of Mechanics and Physics of Solids 21, $395-410$.

Shterenlikht, A. [2003] 3D CAFE modelling of transitional ductile-brittle fracture in steels, $\mathrm{PhD}$. Thesis, Department of Mechanical Engineering, University of Sheffield.

Simo, J. C. and Ortiz, M. [2004] "A unified approach to finite deformation elastoplastic analysis based on the use of hyperelastic constitutive equations," Computer Methods in Applied Mechanics and Engineering 49, 221-245.

Soyarslan, C., Gulcimen, B., Bargmann, S. and Hähner, P. [2015] "Modeling of fracture in small punch tests for small- and large-scale yielding conditions at various temperatures," International Journal of Mechanical Sciences.

Tvergaard, V. and Needleman, A. [1984] "Analysis of the cup-cone fracture in a round tensile bar," Acta Metallurgica 32, 157-169.

Tvergaard, V. [1981] "Influence of voids on shear band instabilities under plane strain conditions," International Journal of Fracture 17, 389-407.

Tvergaard, V. [1982a] "On localization in ductile materials containing spherical voids," International Journal of Fracture 18, 237-252.

Tvergaard, V. [1982b] "Influence of void nucleation on ductile shear fracture at a free surface," Journal of Mechanics Physics of Solids 30, 399-425.

Volokh, K. Y. [2004] "Nonlinear elasticity for modeling fracture of isotropic brittle solids," Journal of Applied Mechanics 71, 141-143.

Volokh, K. Y. [2007] "Hyperelasticity with softening for modeling materials failure," Journal of the Mechanics and Physics of Solids 55, 2237-2264.

Volokh, K. Y. [2015] "Non-linear thermoelasticity with energy limiters," International Journal of Non-Linear Mechanics 76, 169-175.

Xia, L. and Fong Shih, C. [1996] "A fracture model applied to the ductile/brittle regime," Journal de Physique IV 6, 363-372. 